\title{
Analytical Error Rate Performance Evaluation of OFDM-QPSK System over Nakagami Distribution
}

\author{
Mukesh Kumar Mishra \\ Department of Electronics \& Communication Engineering \\ National Institute of Technology Jalandhar, India
}

\begin{abstract}
Modern wireless communication system demands high data rate with acceptable error rate transmission and reception. OFDM a multi-carrier modulation technique will provide the solution for next generation wireless communication, so that it has been employed in numerous wireless standards. This paper presents new technique for the error rate of OFDM-QPSK system over Nakagami-m and Nakagami-n (Rice) fading channels, using characteristics function (CHF) based approach. To derive the SER expression for OFDM-QPSK system, exact PDF in integral form is utilized. Further the average SER have expressed in terms of the exponential and confluent hyper geometric functions.
\end{abstract}

\section{Keywords}

OFDM, Nakagami-m Distribution, Nakagami-n (Rice) Distribution, SER, Multipath Channel.

\section{INTRODUCTION}

Orthogonal Frequency Division Multiplexing (OFDM) is a multi-carrier modulation scheme which is useful to remove the frequency selectivity of the channels because transmitted signals are orthogonal in nature [1]. This special multi carrier technique is based on the fact that the high bit stream data is transmitted over great number of sub-carriers; each has a different frequency and overlapped to each other. Basically this scheme is a combination of multiplexing and modulation which is effectively utilizes to eliminate the need of complex equalization at the receiver side [1-3].

Signal fading, which is due to the multipath propagation, is a major problem in wireless communication [4]. The rapid change of the instantaneous received power due to multipath propagation are generally described with Rayleigh, Rician, Nakagami-m or Nakagami-q model [5-6]. Among different fading model Nakagami-m distribution is a special fading distribution, because of its wide range and simple manipulation [3-7]. Nakagami-n (Rice) and Nakagami-q (Hoyt) are other important fading distributions, but due to complex nature of their probability distribution function, they not received as much importance as the Nakagami-m and Rayleigh distribution, despite the fact that these models exhibit an excellent fit to experimental fading channel measurements for land, mobile, terrestrial, and satellite telecommunications[6-9].

Basically the complexity of the performance evaluation of the system depends on the employed digital-modulation mapping technique and the used fading model. The general modulation method which is employed for the OFDM is either M-phase shift keying (PSK) or M-quadratic amplitude modulation [10]. In [4] used the characteristics function approach to evaluate the BER performance and outage probability over Nakagami-m and Rician fading channel and summarized the advantages of this approach over MGF technique. In [6] utilize PDF method and MGF approach to analyze the system performance over different fading distribution. In [3] Nakagami-m distributed frequency domain samples are approximated by other fading parameter, which is different from previous time domain parameter.

According to[11] in case, if we consider an N-point FFT to determine the sampled frequency domain response from sampled time domain, then for Rayleigh fading the signal samples will have a joint complex Gaussian distribution. Based on this theory it can be concluded that if the time-domain signal is Rayleigh faded then its frequency response will be Rayleigh distribution itself, which is not the case with Nakagami-m distributed envelope.

In digital communication system the error rate has been very extensively used as a performance measurement. Generally researchers to derive the error rate expression utilize the probability density function method or the moment generating function (MGF). In [6,12] to make a common approach for analyzing the error rate performance of the system over different fading channels alternative representation of Gaussian Qfunction is used to express the conditional error probability.

In this paper, we have derived error rate expressions for OFDM system over Nakagami-m and Nakagami-n (Rice) fading channels using characteristic function (CHF) approach. The proposed $\mathrm{CHF}$ method utilizes to evaluate the error rate performance evaluation over different modulation techniques and all generally used diversity techniques within a single common platform.

To derive the SER expression for OFDM-QPSK system over Nakagami distribution, exact PDF of first order is utilized. We have expressed the average error rates in terms of confluent hyper geometric function and exponential function.

The remaining paper planned as follows. The section 2 concerns with the system model. In section 3 we derive an integral expression for the PDF of Nakagami-m and Nakagami-n (Rice) random vectors using CHF approach. In section 4 derived PDF is utilized to derive the SER expressions for QPSK-OFDM system over frequency selective Nakagami-m and Nakagami-n (Rice) fading channels. The section 5 discusses about the graphical results and finally section 7 concludes the work. 


\section{SYSTEM MODEL}

Let we have an OFDM system with $\mathrm{N}$ subcarriers and assume that the data symbol as y (0), y (1)... y (N-1) transmitted over the $\mathrm{N}$ sub carriers are mutually independent; the transmitted equivalent baseband OFDM signal can be expressed as [11]:

$$
s(n)=\frac{1}{N} \sum_{k=0}^{N-1} y(k) \exp \left(\frac{j 2 \pi k n}{N}\right), n=0,1 \ldots N-1
$$

The impulse response of a multipath fading channel is according to [3] model as $\left|h_{i}(n) e^{-j \theta_{i}(n)}\right|$.Here we assume that frequency synchronization is achieved at the receiver side so $r(n)$ can be represented as [13]:

$$
R(k)=y(k) H(k)+W
$$

Where $W$ is an independent identically distributed (i.i.d) Gaussian noise with zero mean and unit variance. The amplitude of $H(k)$ is described as a Nakagami RV with PDF.

\section{CALCUlation OF ENVELOPE PDF}

In this section by utilizing CHF method, we derive the integral expression for the first order PDF of Nakagami-m and Nakagami-n (Rice) distributions.

\subsection{PDF of Nakagami-m RV}

Here Nakagami-m random phase vector is defined to have its amplitude following a Nakagami distribution and its phase following a uniform distribution.

The Nakagami-m distribution is given by [6-7]:

$$
f(r)=\frac{2 m^{m} r^{2 m-1}}{\Gamma(m) \Omega^{m}} e^{\frac{-m r^{2}}{\Omega}}, r \geq 0
$$

The Characteristics function of RV $\mathrm{X}$ is mathematically defined as [14]:

$$
\Psi_{X_{l}}(\alpha)=\mathrm{E}[\exp (\mathrm{j} \alpha \mathrm{X})]
$$

The standard mathematical definitions for finding characteristics function from a PDF for independent random phase vectors are given by [15]:

$$
\Psi_{X l}(\alpha)=\int_{0}^{\infty} f(r) J_{0}(\alpha r) d r
$$

Where $\mathrm{J}_{0}($.$) is the zeroth order Bessel function of the first kind$ [16].

Using the eq. (3), eq. (5) can be written as:

$$
\Psi_{X_{l}}(\alpha)=\int_{0}^{\infty} \frac{2 m^{m} r^{2 m-1}}{\Gamma(m) \Omega^{m}} e^{\frac{-m r^{2}}{\Omega}} J_{0}(\alpha r) d r
$$

To simplify the eq. (6) we use integral identity [17, eq. 6.631.1] and eq. (6) can be expressed as:

$$
\Psi_{X l}(\alpha)={ }_{1} F_{1}\left(m_{l} ; 1 ; \frac{-\Omega_{l}}{m_{l}} \alpha^{2}\right)
$$

Where ${ }_{1} \mathrm{~F}_{1}(., ; ;)$ is confluent hyper geometric function [16]. Equation (7) is the CHF which is same as derived in [15] for Nakagami-m fading channel.
Because channel tap coefficients are independent so joint CHF can be given as [11]:

$$
\Psi_{X l}(\alpha)=\prod_{0}^{M-1}{ }_{1} F_{1}\left(m_{l} ; ; ; \frac{-\Omega_{l} \alpha^{2}}{m_{l}}\right) \approx \Psi(\alpha)
$$

Using inverse Fourier Transform the PDF for RV $X$ is given by [15]:

$$
f_{X}(x)=\frac{1}{2 \pi} \int_{-\infty}^{\infty} e^{-j \alpha x} \Psi(\alpha) d \alpha
$$

After some simplification eq. (9) can be written as [15]:

$$
f_{X}(x)=\frac{1}{\pi} \int_{0}^{\infty} \cos (\alpha x) \Psi(\alpha) d \alpha
$$

Using eq. (8) the integral PDF is given by:

$$
f_{X}(x)=\frac{1}{\pi} \int_{0}^{\infty} \prod_{0}^{M-1}{ }_{1} F_{1}\left(m_{l} ; 1 ; \frac{-\Omega_{l} \alpha^{2}}{m_{l}}\right) \cos (\alpha x) d \alpha
$$

Equation (11) is the expression for the PDF in a integral form,that is further used in error rate calaculation.

\subsection{PDF of Nakagami-n RV}

The Nakagami-n (Rice) distribution is given by [6-7]:

$$
\begin{array}{r}
f(r)=\frac{2(1+K)}{\Omega} r e^{\left(-K^{-(1+K) r^{2} / \Omega}\right)} \\
I_{0}\left(2 r \sqrt{\frac{K(1+K)}{\Omega}}\right), r \geq 0
\end{array}
$$

Using the eq. (12), eq. (5) can be written as

$$
\begin{gathered}
\Psi_{X l}(\alpha)=\int_{0}^{\infty} \frac{2(1+K)}{\Omega} r e^{\left(-K^{-(1+K) r^{2} / \Omega}\right)} I_{0}\left(2 r \sqrt{\frac{K(1+K)}{\Omega}}\right) \\
J_{0}(\alpha r) d r
\end{gathered}
$$

To simplify the eq. (13) we use integral identity [17, eq. 6.633.4] and eq. (13) can be expressed as:

$\Psi_{X l}(\alpha)=\exp \left(\frac{-\Omega_{l}}{4\left(1+K_{l}\right)} \alpha^{2}\right) J_{0}\left(\alpha \sqrt{\frac{K_{l} \Omega_{l}}{\left(1+K_{l}\right)}}\right)_{(14)}$

Because channel tap coefficients are independent so joint $\mathrm{CHF}$ can be given as [11]:

$$
\begin{aligned}
\Psi_{X l}(\alpha)= & \prod_{0}^{M-1} \exp \left(\frac{-\Omega_{l}}{4\left(1+K_{l}\right)} \alpha^{2}\right) \\
& J_{0}\left(\alpha \sqrt{\frac{K_{l} \Omega_{l}}{\left(1+K_{l}\right)}}\right) \approx \Psi(\alpha)
\end{aligned}
$$

Using eq. (10) the integral PDF is given by: 


$$
\begin{gathered}
f_{X}(x)=\frac{1}{\pi} \int_{0}^{\infty} \cos (\alpha x) \exp \left(\frac{-\Omega_{l}}{4\left(1+K_{l}\right)} \alpha^{2}\right) \\
J_{0}\left(\alpha \sqrt{\frac{K_{l} \Omega_{l}}{\left(1+K_{l}\right)}}\right) d \alpha
\end{gathered}
$$

Equation (16) is the derived integral expression for PDF, which is same as derived in [18] for BPSK system.

\section{ERROR RATES ANALYSIS USING SINGLE-CHANNEL RECEPTION}

\subsection{SER Derivation for Nakagami-m fading Channel}

The conditional error probability (CEP) $[6,12]$ for particular modulation is given by $\mathrm{Q}(\mathrm{x})$ where $Q(x)=\frac{1}{\sqrt{2 \pi}} \int_{x}^{\infty} e^{\left(\frac{-t^{2}}{2}\right)} d t$.Using the alternative representation of above equation as given in [13].

$$
Q(S x)=\frac{1}{\pi} \int_{0}^{\frac{\pi}{2}} e^{\left(\frac{-S^{2} x^{2}}{2 \sin ^{2}(\phi)}\right)} d \phi
$$

In many research papers the symbol error rate for QPSK is given by:

$$
=\operatorname{erfc}(\sqrt{\gamma})-.25 \operatorname{erfc}^{2}(\sqrt{\gamma})
$$

Using the relationship between Q-function and erfc function [19, pp.649, eq. (F.12)] we can approximate eq. (18) as follows:

$$
\approx 2 Q(S x)
$$

In above eq. (19) $\mathrm{S}$ represents the symbol to noise ratio for the OFDM-QPSK system.

Averaging the CEP over derived PDF the error rate denoted by $\mathrm{P}_{\mathrm{e}}(\mathrm{S})$ can be expressed as [11]:

$$
\begin{aligned}
P_{e}(S)= & \int_{0}^{\infty} 2 Q(S x) f_{X}(x) d x \\
P_{e}(S)= & \frac{2}{\pi^{2}} \int_{0}^{\infty} \Psi(\alpha) d \alpha \\
& \left(\int_{0}^{\pi / 2} \int_{0}^{\infty} \cos (\alpha x) \exp \left(\frac{-S^{2} x^{2}}{2 \sin ^{2}(\phi)}\right) d x\right) d \phi
\end{aligned}
$$

Using the identity $[17,3.896 .4]$ eq. (21) can be simlified as:

$$
\begin{aligned}
& P_{e}(S)=\frac{2}{\pi^{2}} \int_{0}^{\infty} \Psi(\alpha) d \alpha \\
& \left(\int_{0}^{\pi / 2} \frac{1}{2} \sqrt{\frac{2 \pi \sin ^{2}(\phi)}{S^{2}}} \exp \left(-\frac{\alpha^{2} \sin ^{2}(\phi)}{2 S^{2}}\right) d \phi\right)
\end{aligned}
$$

$$
\begin{gathered}
P_{e}(S)=\frac{1}{\pi} \int_{0}^{\infty} \Psi(\alpha) e^{\frac{-\alpha^{2}}{2 S^{2}}} \frac{\operatorname{erfi}\left(\frac{\alpha}{\sqrt{2 S}}\right)}{\alpha} d \alpha \\
P_{e}(S)=\frac{1}{\pi} \int_{0}^{\infty} \prod_{0}^{M-1}{ }_{1} F_{1}\left(m_{l} ; 1 ; \frac{-\Omega_{l} \alpha^{2}}{m_{l}}\right) \\
e^{\frac{-\alpha^{2}}{2 S^{2}}} \frac{\operatorname{erfi}\left(\frac{\alpha}{\sqrt{2 S}}\right)}{\alpha} d \alpha
\end{gathered}
$$

Equation (24) is used to express the SER expression for the single tap and two tap multipath channels. For single tap BER expression can be given as:

$$
\begin{aligned}
& P_{e}(S)=\frac{1}{\pi} \int_{0}^{\infty} F_{1}\left(m_{0} ; 1 ; \frac{-\Omega_{0} \alpha^{2}}{m_{0}}\right) \\
& e^{\frac{-\alpha^{2}}{2 S^{2}}} \frac{\operatorname{erfi}\left(\frac{\alpha}{\sqrt{2 S}}\right)}{\alpha} d \alpha(25) \\
& P_{e}(S)=\frac{1}{\pi} \int_{0}^{\infty} F_{1}\left(m_{0} ; 1 ; \frac{-\Omega_{0} \alpha^{2}}{m_{0}}\right) \\
& e^{\frac{-\alpha^{2}}{2 S^{2}}} \frac{\operatorname{erfi}\left(\frac{\alpha}{\sqrt{2 S}}\right)}{\alpha} d \alpha(26)
\end{aligned}
$$

In the similar manner for the two tap multipath channel we have expression as:

$$
\begin{array}{r}
P_{e}(S)=\frac{1}{\pi} \int_{0}^{\infty} F_{1}\left(m_{0} ; 1 ; \frac{-\Omega_{0} \alpha^{2}}{m_{0}}\right){ }_{1} F_{1}\left(m_{1} ; 1 ; \frac{-\Omega_{1} \alpha^{2}}{m_{1}}\right) \\
e^{\frac{-\alpha^{2}}{2 S^{2}}} \frac{\operatorname{erfi}\left(\frac{\alpha}{\sqrt{2 S}}\right)}{\alpha} d \alpha(27)
\end{array}
$$

\subsection{SER Derivation for Nakagami-n (Rice) Fading Channel}

Using eq. (16) and (20) the error rate expression for QPSK system is given as:

$$
\begin{aligned}
P_{e}(S)= & \frac{2}{\pi^{2}} \int_{0}^{\infty} \Psi(\alpha) d \alpha \\
& \left(\int_{0}^{\pi / 2} \int_{0}^{\infty} \cos (\alpha x) \exp \left(\frac{-S^{2} x^{2}}{2 \sin ^{2}(\phi)}\right) d x\right) d \phi(28)
\end{aligned}
$$

Using the identity [17,eq. 3.896 .4$]$ eq. (28) can be simlified as:

$$
P_{e}(S)=\frac{2}{\pi^{2}} \int_{0}^{\infty} \Psi(\alpha) d \alpha
$$

After integration and some simplification eq. (22) can be expressed as: 


$$
\left(\int_{0}^{\pi / 2} \frac{1}{2} \sqrt{\frac{2 \pi \sin ^{2}(\phi)}{S^{2}}} \exp \left(-\frac{\alpha^{2} \sin ^{2}(\phi)}{2 S^{2}}\right) d \phi\right)(29
$$

After integration and some simplification eq. (29) can be expressed as:

$$
\begin{gathered}
P_{e}(S)=\frac{1}{\pi} \int_{0}^{\infty} \Psi(\alpha) e^{\frac{-\alpha^{2}}{2 S^{2}}} \frac{\operatorname{erfi}\left(\frac{\alpha}{\sqrt{2 S}}\right)}{\alpha} d \alpha \\
P_{e}(S)=\frac{1}{\pi} \int_{0}^{\infty} \prod_{0}^{M-1} \exp \left(\frac{-\Omega_{l}}{4\left(1+K_{l}\right)} \alpha^{2}\right) J_{0}\left(\alpha \sqrt{\frac{K_{l} \Omega_{l}}{\left(1+K_{l}\right)}}\right) \\
e^{\frac{-\alpha^{2}}{2 S^{2}}} \frac{\operatorname{erfi}\left(\frac{\alpha}{\sqrt{2 S}}\right)}{\alpha} d \alpha(31)
\end{gathered}
$$

Equation (31) is used to express the SER expression for the single tap and two tap multipath channels. For single tap SER expression can be given as:

$$
\begin{array}{r}
P_{e}(S)=\frac{1}{\pi} \int_{0}^{\infty} \exp \left(\frac{-\Omega_{0}}{4\left(1+K_{0}\right)} \alpha^{2}\right) J_{0}\left(\alpha \sqrt{\frac{K_{0} \Omega_{0}}{\left(1+K_{0}\right)}}\right) \\
e^{\frac{-\alpha^{2}}{2 S^{2}}} \frac{\operatorname{erfi}\left(\frac{\alpha}{\sqrt{2 S}}\right)}{\alpha} d \alpha
\end{array}
$$

In the similar manner for the two tap multipath channel we have expression as:

$$
\begin{aligned}
& P_{e}(S)=\frac{1}{\pi} \int_{0}^{\infty} \exp \left(\frac{-\Omega_{0}}{4\left(1+K_{0}\right)} \alpha^{2}\right) J_{0}\left(\alpha \sqrt{\frac{K_{0} \Omega_{0}}{\left(1+K_{0}\right)}}\right) \times \\
& \exp \left(\frac{-\Omega_{1}}{4\left(1+K_{1}\right)} \alpha^{2}\right) J_{0}\left(\alpha \sqrt{\frac{K_{1} \Omega_{1}}{\left(1+K_{1}\right)}}\right) e^{\frac{-\alpha^{2} S^{2}}{\operatorname{erfi}\left(\frac{\alpha}{\sqrt{2 S}}\right)}} d \alpha
\end{aligned}
$$

When $\Omega_{0}=\Omega_{1}=\Omega$ and $K_{0}=K_{1}=K$ eq. (33) is given by:

$$
\begin{array}{r}
P_{e}(S)=\frac{1}{\pi} \int_{0}^{\infty} \exp \left(\frac{-\Omega}{2(1+K)} \alpha^{2}\right)\left(J_{0}\left(\alpha \sqrt{\frac{K \Omega}{(1+K)}}\right)\right)^{2} \\
e^{\frac{-\alpha^{2}}{2 S^{2}}} \frac{\operatorname{erfi}\left(\frac{\alpha}{\sqrt{2 S}}\right)}{\alpha} d \alpha(34)
\end{array}
$$

\section{RESULTS AND DISCUSSION}

In this section,we present a critical discussion on error rates performance of OFDM-QPSK system using above derived analytical expression.

\section{Case 5.1: Under the influence of Nakagami-m fading} channel

In this section the error rate performance of an OFDM system over Nakagami-m fading channel is analytically evaluated. By varying the fading parameter $m$ obtaining the BER vs $\mathrm{SNR}$ is plotted as shown in Fig. 1 for single tap channel. Fig. 2 for two tap channel with $\mathrm{m}_{0}=\mathrm{m}_{1}=\mathrm{m}$ and $\Omega_{0}=\Omega_{1}=1$. As increasing the fading parameter $\mathrm{m}$, SER starts decreasing as expected for single tap. This fact is already reported in many research papers for Nakagami-m fading channels [2-3]. When we consider two tap then for the Nakagami-m channel, slopes of the error rate performance increases if fading parameter $\mathrm{m}$ increases. Further, if we increase $\mathrm{m}$, no reduction in SER has been reported rather it starts increasing. So this proves that we cannot increase the value of fading parameter $\mathrm{m}$ beyond a limit. Here the threshold value for Nakagami-m is achieved to be 1.4 through analytical results.

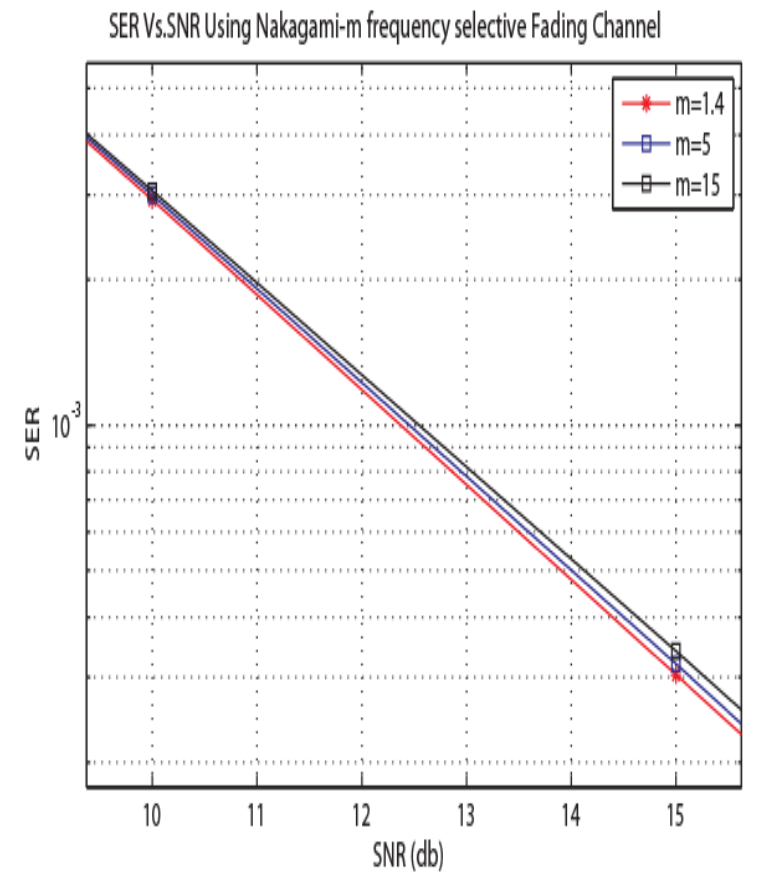

Fig.1 SER Vs SNR for OFDM-QPSK system with single tap 


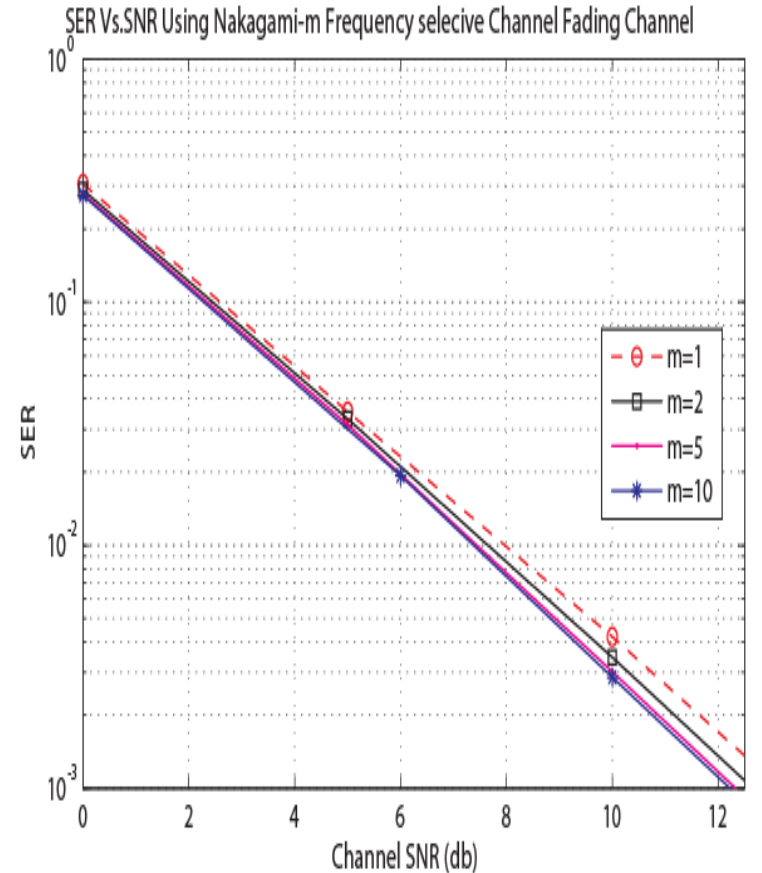

Fig.2 SER Vs SNR for OFDM-QPSK system with two tap

\section{Case 5.2: Under the influence of Nakagami-n (Rice) fading channel}

In this section,we present a discussion on error rates performance of OFDM-QPSK system. By varying the fading parameter $n$ obtaining the SER vs SNR is plotted as shown in Fig. 3 for single tap channel. Fig. 4 for two tap channel with $\mathrm{n}_{0}=\mathrm{n}_{1}=\mathrm{n}$ and $\Omega_{0}=\Omega_{1=} \Omega=1$.

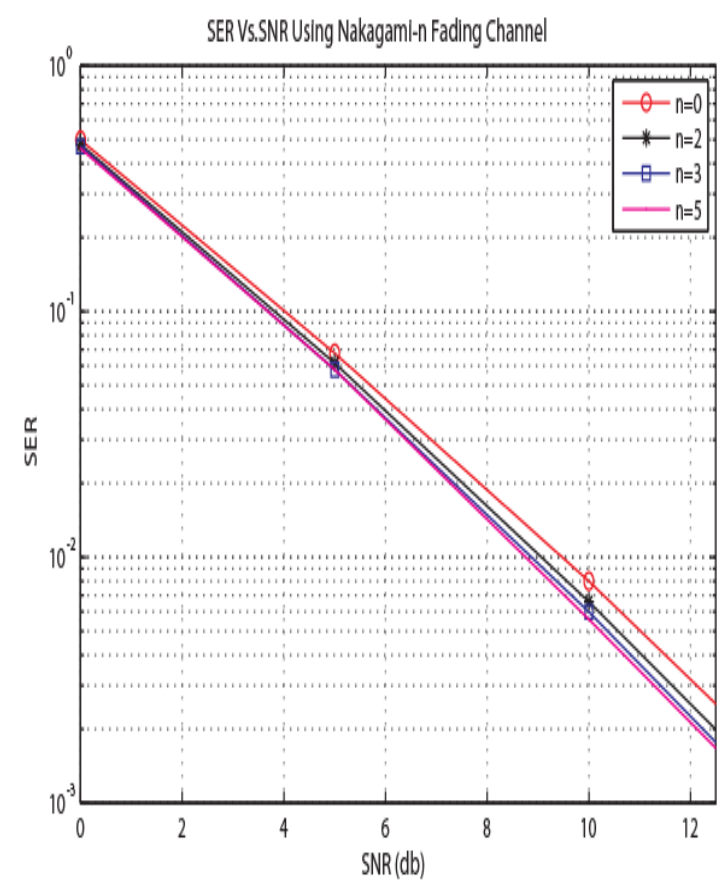

Fig.3 SER Vs SNR for OFDM-QPSK system with single tap

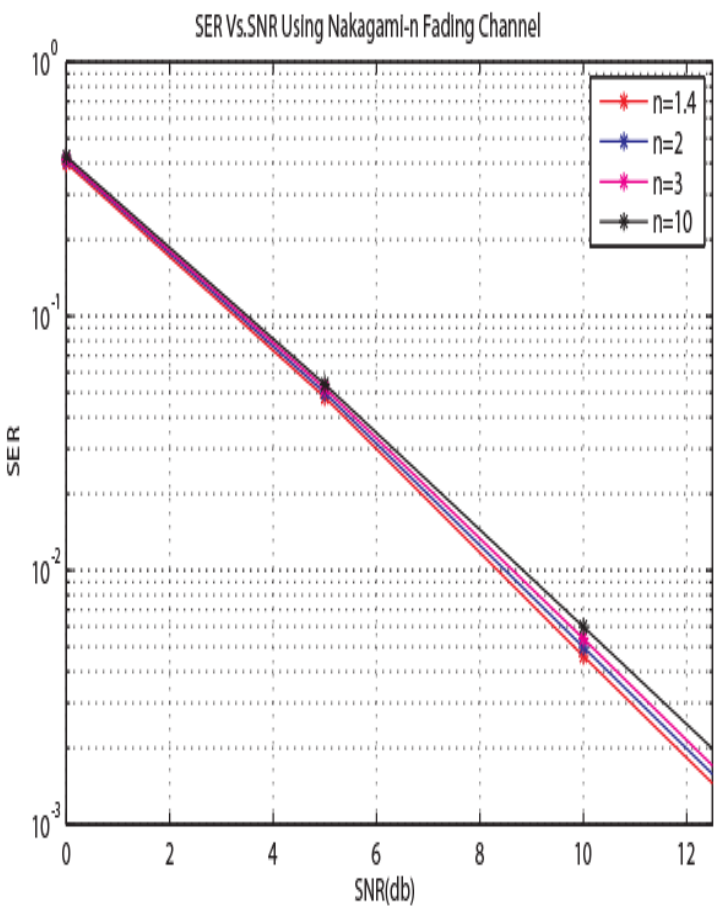

Fig.4 SER Vs SNR for OFDM-QPSK system with two tap

If fading parameter $\mathrm{n}$ increases, slopes of the error rate performance increases. But further, if we increase $n$, no reduction in SER has been reported rather it starts increasing. So this put a limit to increase the value of $n$ beyond the certain value. This fact is expected because according to [6]-[9] we can approximate the Nakagami-n (Rice) distribution by a Nakagami$m$ distribution that means if for the Nakagami-m (Two Tap) case error rate performance degrades with increasing values of $\mathrm{m}$ as reported in [1],[11],[20] then same nature of the SER performance should be shown by the Nakagami-n (Rice) fading distribution, and same results, we are achieved in our analysis. Here the threshold value for Nakagami-n is achieved to be 1.4 through analytical results.

\section{CONCLUSION}

In this paper average error rate performance of OFDM system over Nakagami-m and Nakagami-n (Rice) fading channel is analyzed. The error rate expressions are derived by utilizing integral PDF. The derived PDF remove the complexity of the SER expression efficiently. Based on this new approach, either numerically or analytically, performance of various modulations over multipath fading channels can be evaluated. Finally it has been found that, depending on the number of channel taps, larger Nakagami-m and Nakagami-n (Rice) fading parameters do not necessarily give smaller error rates. 


\section{REFERENCES}

[1] Neetu Sood, Ajay K Sharma, Moin Uddin, "BER Performance of OFDM-BPSK and -QPSK over Generalized Gamma Fading Channel", International Journal of Computer Applications (IJCA), vol. 3, no.6, pp.13-16, June 2010.

[2] Ramjee Prasad, "OFDM for Wireless Communications Systems", Artech House, 2004.

[3] Zhengjiu Kang, Kung Yao, and Flavio Lorenzelli, "Nakagami-m fading modeling in the frequency domain for OFDM system analysis", IEEE Commun. Lett. vol. 7, no. 10, pp. 484-486, Oct. 2003.

[4] A. Annamalai, C. Tellambura, And Vijay K. Bhargava, "A General Method For Calculating Error Probabilities Over Fading Channels", IEEE Trans. on Commun., vol. 53, no. 5, pp.841-852, May 2005.

[5] J. G. Proakis, "Digital Communications", McGraw-Hill, 1995.

[6] Marvin K. Simon, and Mohamed-Slim Alouini, "Digital Communication over Fading Channels: A Unified Approach to Performance Analysis", John Willy \& Sons, 2000.

[7] M. Nakagami, "The m-distribution, a general formula of intensity distribution of rapid fading in Statistical Methods in Radio Wave Propagation", W. G. Hoffman, Oxford, England, Pergamum, 1960.

[8] Dimitris A. Zogas, George K. Karagiannidis, and Stavros A. Kotsopoulos, "Equal Gain Combining over Nakagami-n (Rice) and Nakagami-q (Hoyt) Generalized Fading Channels", IEEE Trans. on Wireless Comm., vol. 4, no. 2,pp.374-379 Mar. 2005.

[9] A. A. Abu-Dayya, N. C. Beaulieu, "Micro diversity on Rician Fading Channels", IEEE Trans. Commun, vol. 42, no. 6, June 1994.

[10] Richard V. Nee, Ramjee Prasad, "OFDM for Wireless Multimedia. Communications", Artech House, 2000.
[11] Z. Du, J. Cheng, N. C. Beaulieu, "Accurate error rate performance analysis of OFDM in frequency-selective Nakagami- $m$ fading channels", IEEE Trans. Commun. vol. 54, no. 2, pp. 319-328 Feb 2006.

[12] Marvin K. Simon, and Mohamed-Slim Alouini "A Unified Approach to the Performance Analysis of Digital Communication over Generalized Fading Channels", Proceedings of IEEE, vol.86, no.9, pp. 1860-1877, Sep. 1998.

[13] Zheng du, Julian Cheng and Norman C. Beaulieu, "Error Rate of OFDM Signals on Frequency Selective Nakagamim Fading Channels", Globecom, vol.06, pp. 3994-3998, Dec. 2004

[14] A. Papoulis, Probability, "Random Variables, and Stochastic Processes", 3rd Ed. New York: McGraw-Hill, 1991.

[15] Norman C. Beaulieu and Julian Cheng, "Precise error rate analysis of bandwidth efficient BPSK in Nakagami fading and co channel interference", IEEE Trans. Commun. vol.52, pp. 149-158, Jan. 2004.

[16] M. Abramowitz and I. A. Stegun, "Handbook of Mathematical Functions with Formulas, Graphs, and Mathematical Tables", New York: Dover, 1972.

[17] I. S. Gradshteyn and I. M. Ryzhik, "Table of Integrals, Series, and Products", 6th ed. San Diego, CA: Academic, 2000 .

[18] Mukesh Kumar Mishra, Neetu Sood, Ajay K Sharma, "New BER Analysis of OFDM System over Nakagami-n (Rice) Fading Channel", International Journal of Computer Science, Engineering and Information Technology (IJCSEIT),vol.1, no.3,pp.12-20, Aug 2011.

[19] Theodore S. Rappaport, "Wireless Communications. Principles and Practice", Prentice-Hall, 2008.

[20] Neetu Sood, Ajay K Sharma, Moin Uddin, "BER Performance of OFDM-BPSK and QPSK over Nakagami$\mathrm{m}$ Fading Channels", Proc. of $2^{\text {nd }}$ IEEE International Advance computing Conference, pp. 88-90, Feb. 2010. 